\title{
Improvement of Spatial Resolution by Selfconsistent Full Muon Track Reconstruction in Gaseous Detectors
}

\author{
Bernhard Flierl $^{* a}$, Otmar Biebel ${ }^{a}$, Maximilian Herrmann ${ }^{a}$, Ralf Hertenberger ${ }^{a}$, Felix \\ Klitzner $^{a}$, Philipp Lösel ${ }^{a}$, Ralph Müller ${ }^{a}$ and Andre Zibell ${ }^{c}$ \\ ${ }^{a}$ Ludwig-Maximilians-Universität München, Germany \\ ${ }^{b}$ Julius-Maximilians-Universität Würzburg, Germany \\ E-mail: Bernhard.Flierl@physik.uni-muenchen.de
}

\begin{abstract}
Full track reconstruction for charged particles in thin gaseous detectors can be achieved using a Time-Projection-Chamber like (TPC) read-out and analysis method. This method has proven to be very successful for thermal neutron detection in gaseous electron multiplier (GEM) detectors[1], based on the full track reconstruction of the charged Helium or Lithium ions produced in a thin ${ }^{10} \mathrm{~B}$ conversion layer building the cathode of the triple GEM detector. An improvement from FWHM $3.4 \mathrm{~mm}$ to $0.25 \mathrm{~mm}$ of the spatial resolution of the interaction point of the neutron in the ${ }^{10} \mathrm{~B}$ layer has been observed using an Ar-CO2 gas mixture as detector gas. For the achievable track resolution the drift velocity and thus the composition of the drift gas is of big importance. A self-consistent algorithm allows for optimized results without the development of gas-parameters, as otherwise usual, in prior test experiments with well known angle of incidence of the ions. Simulations predict, that by application of this method the spatial resolution for minimal ionizing particles can be improved as well. For verification a compact cosmic muon telescope has been commissioned, which consists of four triple GEM detectors with two-dimensional strip read-out of $0.4 \mathrm{~mm}$ pitch in $\mathrm{x}$ and $\mathrm{y}$. All strips are read out by APV25 frontend boards [2]. Muon tracks are reconstructed using the TPC-like method in one of the detectors and are then compared to the predicted track from the other three detectors defined by the center of charge position in every detector.
\end{abstract}

38th International Conference on High Energy Physics

3-10 August 2016

Chicago, USA

\footnotetext{
* Speaker.
} 


\section{Motivation}

Spatial resolution in planar gaseous detectors is in many cases dependent on the inclination angle of the track of an ionizing particle with respect to the read-out plane. The quality of the tracks, identified by electron ion-pairs in the active volume of the detector, has large influence on its position reconstruction capabilities. A standard analysis would determine the position of a hit in the detector by averaging over all read-out segments weighted with the collected charge, which is called the centroid position. This works well for particles traversing the detector perpendicular to its read-out plane and best if the charge production along the track is homogeneous. The here investigated GEM-detectors for example have shown a spatial resolution of $100 \mu \mathrm{m}$ (see [4]).

In the following an approach towards a full-track based reconstruction is proposed in order to increase the spatial resolution particularly for non-homogeneously ionizing particles (compare figure 1). This has been studied using with cosmic muons ${ }^{1}$. This method has been proven useful for particles, which stop inside the active gaseous volume of a detector (see [4]).

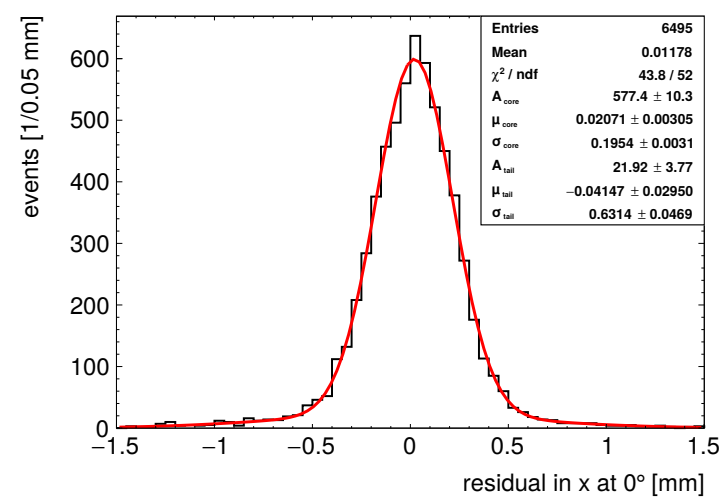

Figure 1: Measured residuals for a triple GEM-detector with cosmic muons of perpendicular incident, determined in a detector telescope with a standard centroid analysis method fitted with a double Gaussian function. The achieved width is $\sigma_{\text {weighted }}=(240 \pm 10) \mu \mathrm{m}$, particularly limited by the strongly clustering energy loss of the muons.

\section{Reconstruction Method}

The main idea of the described method is to compensate for the charge clustering due to nonhomogeneous ionization in the drift volume of the detector. Generic events with homogeneous and clustered charge distribution are shown in figure 2. The method described here is generically applicable, but will be discussed exemplarily on detectors with strip read-out.

The centroid of a track is defined to be the hit position in precision direction of the detector at some fixed distance to the read-out structure. In the simplest case this is the distance to the center of the drift gap. If a particle would stop in the detector the assumption of the position to be at the center of the drift gap doesn't hold anymore. Nonetheless the origin of the track still has to be close to the cathode of the detector. This can be used to trail the track in the drift gap back to its

\footnotetext{
${ }^{1}$ Cosmic muons have a broad energy distribution with $\left\langle E_{m u}\right\rangle \approx 4 \mathrm{GeV}$ at sea-level [3]
} 

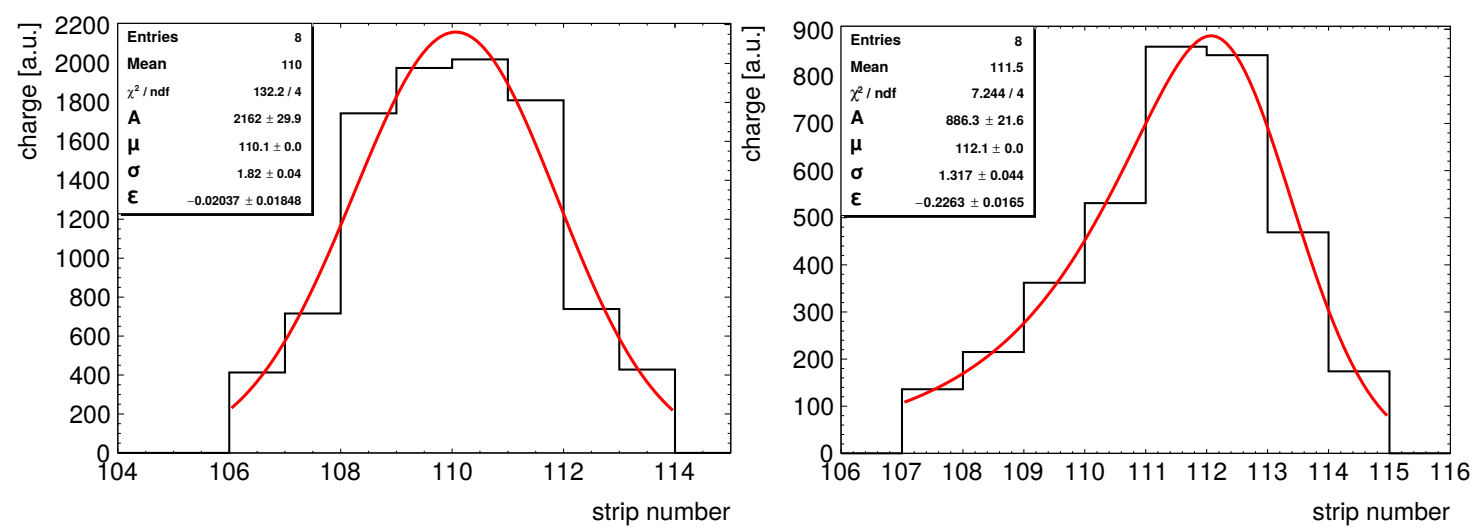

Figure 2: Charge distribution on strips in one read-out direction for a homogeneously charged track (left) and a skewed track due to clustering (right) fitted with eq. [4.1]. The centroid position and the geometric mean deviate by $0.2 \mathrm{~mm}$ for the skewed distribution

start $\vec{x}_{C}$, where the position in $\mathrm{Z}$ is known. Due to possible charge coupling on the read-out pattern and lateral diffusion in the drift as well as in the amplification stage the position of this point in the precision directions can usually not be obtained directly by determination of the edges of the charge distribution. This can be overcome by an extrapolation from the centroid position $\vec{x}_{\text {cent }}$ in the following way:

$$
\vec{x}_{C}=\left(\begin{array}{l}
x_{\text {cent }} \\
y_{\text {cent }} \\
z_{\text {cent }}
\end{array}\right)+\lambda \frac{l_{0}}{2}\left(\begin{array}{c}
\cos \phi \sin \theta \\
\sin \phi \sin \theta \\
\cos \theta
\end{array}\right)
$$

Where $l_{0}$ is the track length inside of the detector, $\phi$ and $\theta$ are the azimuthal and polar angles of the track, $\lambda$ denotes the necessary fraction of the track in the detector to reach the cathode. In the perfectly homogeneous case $\lambda$ would be -1 , in reality it reaches values in the range $[-2,0]$.

Depending on the detector and drift gas, the measured values have to be modified in order to get the related track parameters. The real track length for example has to be obtained by considering a minimal measured track length due to lateral diffusion of the electron cloud in the detector. This leads to a minimal cluster radius of $d_{0}$ and therefore: $l_{\text {real }}=\sqrt{l_{\text {meas. }}^{2}-d_{0}^{2}}$ For a strip read-out it is natural to determine the projected track-length $l_{\text {proj }}$ independently in both read-out directions, which for X-direction can be written as:

$$
l_{\text {proj }}=l_{0} \cos \phi \sin \theta=l_{0} \sin \theta_{\text {proj }}
$$

In the following always projections in one of the read-out directions are used. The value of $\lambda$ can be obtained from the charge skewness along the read-out direction $\varepsilon$.

$$
\lambda=\operatorname{sign}(\theta)(1-\varepsilon)
$$

Which then yields:

$$
x_{C}=x_{c e n t}+a \cdot \operatorname{sign}(\theta) \cdot(1-\varepsilon)\left(\sqrt{\left(\frac{l_{p r o j}}{b}\right)^{2}-d_{0}^{2}}\right)
$$


The centroid position is corrected depending on the inclination of the track $\theta$, the projected track length $l_{p r o j}$ and the skewness of the charge distribution $\varepsilon$ separately in both read-out directions. The parameters $a$ and $b$ are detector and normalization parameters, which are obtained by fits to the epsilon and $l_{\text {proj }}$ distribution.

\section{Experimental Setup}

The method was tested with a cosmic muon telescope consisting of four triple GEM-detectors, as shown in figure 3 . The position measured by the third detector was compared to a track reference defined by the other three detectors. The distances between the detectors were chosen to minimize the track uncertainty at the position of the test-detector and the overall height of the telescope was minimized in order to obtain a sufficient muon rate, which was overall $0.1 \mathrm{~Hz}$. Three of the detectors were equipped with low material budget read-out boards and all had $10 \mu \mathrm{m}$ thin Kapton windows in order to minimize straggling of the muons in the detector system. The interior of the detectors was identical with an active volume of $(100 \times 100 \times 6) \mathrm{mm}^{3}$ and three layers of $60 \mu \mathrm{m}$ thick GEM-foils. As drift gas an Ar- $\mathrm{CO}_{2}$ mixture of 93:7 vol\% was used and the pressure inside the detectors was fixed to $(990 \pm 5)$ mbar. The read-out anode of all detectors consisted of crossed strips with a pitch of $0.4 \mathrm{~mm}$. The strips were equipped with time resolving read-out electronics based on APV25 chips[2] and the SRS DAQ-system [5].

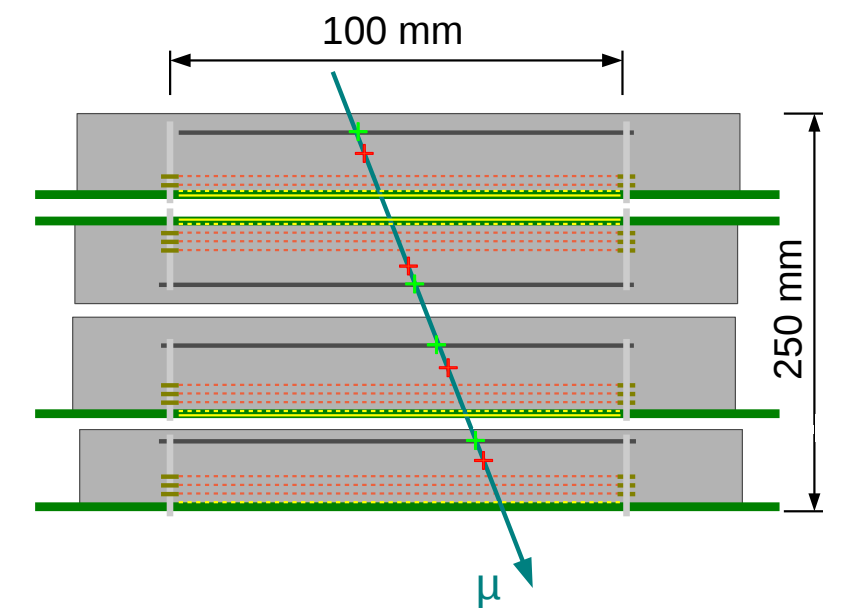

Figure 3: Schematic of the muon telescope with three reference triple GEM-detectors and the test-detector at the second to lowest position

\section{Track Reconstruction in a single detector}

The projected track length in both read-out directions and the asymmetry of the charge distribution were determined by a fit of a piece wise defined Gaussian fit (as previously shown in figure 2):

$$
q(x)=A \cdot \exp \left(\left(\frac{x-\mu}{2 \cdot(\sigma+H(\mu)) \cdot \varepsilon \cdot(x-\mu)}\right)^{2}\right)
$$


$\varepsilon$ is a measure for the skewness of the charge distribution and the projected track length is obtained by definition of: $l_{\text {proj }} \triangleq \sigma \cdot(1+\varepsilon)$. This value is proportional to the number of strips hit, but minimizes the effect of discrete values for the track length.

The projected track inclination $m$ is determined by the start time of the signal on every strip and by a linear fit to the distribution of these timings as a function of the strip position, which is shown in figure 4 (left). The parameters $d_{0}$ and $b$ can be obtained by a fit to the frequency distribution of $l_{\text {proj }}$ by extrapolation to the lowest value (compare fig. 4 (right)) and by comparison of $l_{\text {proj }}$ with the number of strips hit respectively.
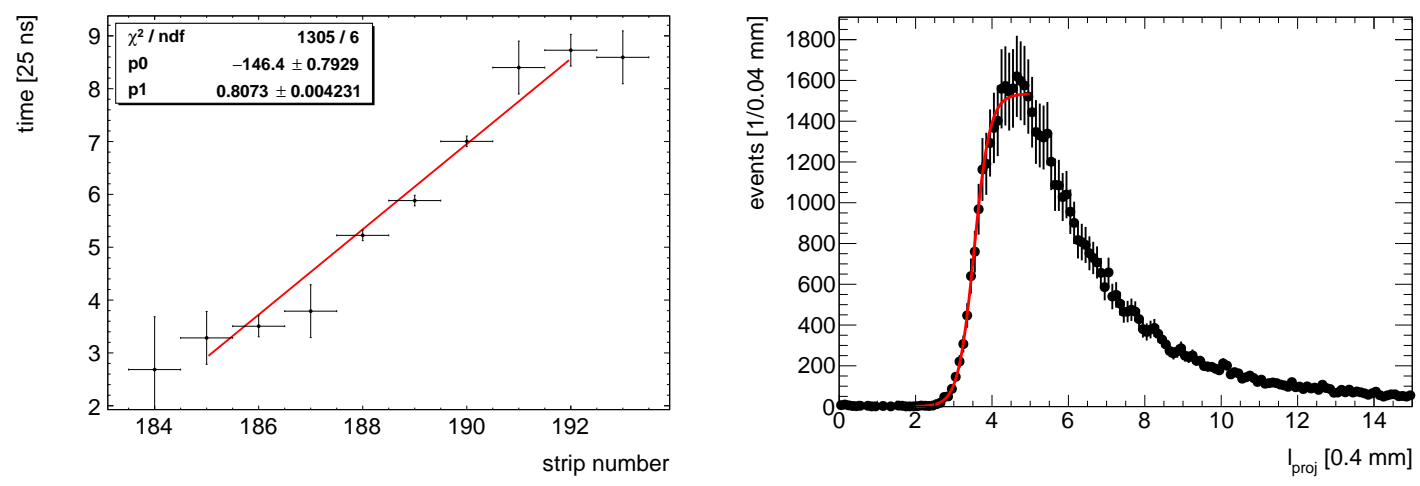

Figure 4: Track angle reconstruction in one read-out direction by fit to drift time distribution on the strips (left), the inclination angle is obtained by the slope with known electron drift velocity. From the distribution of projected track lengths in one read-out direction (right) minimal cluster size $d_{0}$ and the normalization factor $b$ are obtained (compare eq. [2.4])

\section{Spatial Resolution}

In order to determine the spatial resolution for the tested detector an approach has been chosen, which does not explicitly need the track resolution of the telescope for the calculation of the detector resolution: $\sigma_{d e t}=\sqrt{\sigma_{e x} \cdot \sigma_{i n c}}$ (see [6]). Where $\sigma_{e x}$ is the residual, if the test-detector is excluded from a track fit through the telescope and $\sigma_{i n c}$ is the residual with the detector included. Both have been determined by a weighted double Gaussian fit. Figure 5 shows the spatial resolution $\sigma_{d e t}$ plotted against the track inclination for the centroid method and the corrected method. As expected the spatial resolution for the centroid method is strongly depending on the track inclination. Compared to perpendicular tracks the spatial resolution is a factor of 3 worse, if tracks with more than 0.36 $\left(=20^{\circ}\right)$ inclination are considered. For inclinations between 0.1 and 0.35 (corresponding to $~$ $\left.(5-15)^{\circ}\right)$ the corrected method improved the spatial resolution up to $0.1 \mathrm{~mm}$. The spatial resolution over all angles enhances from $(290 \pm 10)$ to $(220 \pm 10) \mu \mathrm{m}$. Due to the limitations in the set-up and low statistics larger incident angles have not yet been investigated.

\section{Conclusion}

The overall spatial resolution of planar gaseous detector can be improved by considering the whole track of a charged particle inside the detector. The application of a TPC-like extrapolation 


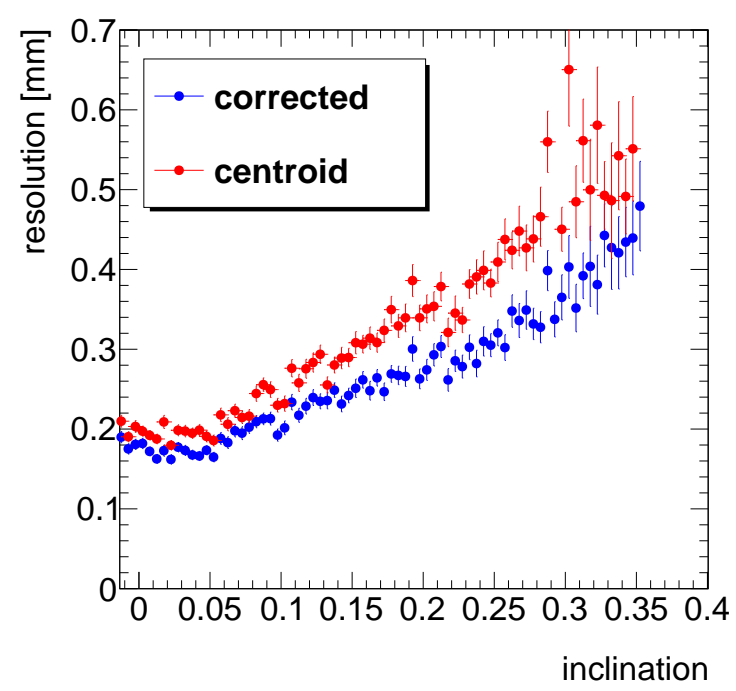

Figure 5: Measured spatial resolution for cosmic muons with a triple GEM detector plotted against the inclination of the muon track. At large inclinations the measurement suffered from the limited acceptance of the muon telescope

algorithm allows for optimized position reconstruction for inclined tracks. The steep inclination dependence of the spatial resolution can be diminished by extrapolation to the start point of the track and consideration of charge distribution along the track. Tests with a cosmic muon telescope showed best improvement of the spatial resolution for inclination angles between 5 and $15^{\circ}$ with an average improvement of about $25 \%$.

\section{References}

[1] F. Sauli, GEM: A new concept for electron amplification in gas detectors, Nucl. Instrum. Meth. A386 (1997) 531-534.

[2] M. French et al.,Design and Results from the APV25, a Deep Submicron CMOS Front-End Chip for the CMS Tracker, Nuclear Instruments and Methods in Physics Research A 466 (2001) 359-365.

[3] K. A. Olive et al. [Particle Data Group Collaboration], Chin. Phys. C 38, 090001 (2014).

[4] B. Flierl et al., TPC-like readout for thermal neutron detection using a GEM-detector, Nuclear Instruments and Methods in Physics Research A 824 (2016) 528-531.

[5] S. Martoiu et al., Development of the scalable readout system for micro-pattern gas detectors and other applications, Journal of Instrumentation 8 (2013) C0301.

[6] R. K. Carnegie et al., Resolution studies of cosmic-ray tracks in a TPC with GEM readout, Nuclear Instruments and Methods in Physics Research A 538 (2005) 372-383. 